\title{
FRACTAL IMAGE FEATURE VECTORS WITH APPLICATIONS IN FRACTOGRAPHY
}

\author{
HyNeK LAUSCHMANN ${ }^{1}$, PETR BEDNAŘíK ${ }^{2}$, ZuZANA SEKEREŠOVÁ ${ }^{1}$ AND MiCHAL BENEŠ ${ }^{2}$ \\ ${ }^{1}$ Department of Materials, Czech Technical University, Faculty of Nuclear Sciences and Physical Engineering, \\ Trojanova 13, Praha 2, 120 00, Czech Republic; ${ }^{2}$ Department of Mathematics, Czech Technical University, \\ Faculty of Nuclear Sciences and Physical Engineering, Trojanova 13, Praha 2, 120 00, Czech Republic \\ e-mail: lausch@kmat.fjfi.cvut.cz \\ (Accepted November 26, 2007)
}

\begin{abstract}
The morphology of fatigue fracture surface (caused by constant cycle loading) is strictly related to crack growth rate. This relation may be expressed, among other methods, by means of fractal analysis. Fractal dimension as a single numerical value is not sufficient. Two types of fractal feature vectors are discussed: multifractal and multiparametric. For analysis of images, the box-counting method for 3D is applied with respect to the non-homogeneity of dimensions (two in space, one in brightness). Examples of application are shown: images of several fracture surfaces are analyzed and related to crack growth rate.
\end{abstract}

Keywords: crack growth rate, fatigue, fractal, fractography, multifractal.

\section{INTRODUCTION}

One of the basic tasks of qualitative fractography is the reconstitution of fatigue crack growth history from the morphology of the crack surface. The merits of all methods consist in relating the morphology of crack surface to the crack growth rate (CGR). As a basis for expressing this relation, fractal analysis may also be used (among many other methods).

The main characteristic of an object from the viewpoint of fractal geometry is a numeric parameterthe fractal dimension. This way of fractal analysis was also applied in fractography, e.g., Wiencek and Czarski, 2000, Mazancová and Mazanec, 2000, Němec et al., 2000. Mecholsky et al., 2002, Mecholsky 2006, Carpinteri et al., 2002 and Carpinteri and Spagnoli, 2004, related fractal dimension to parameters of fracture mechanics. In these cases, application of fractal dimension led to valuable results.

Sekerešová (2004) studied the relation between fractal dimension of images of fracture surfaces and fatigue crack growth rate. The relationships did not prove to be sufficiently tight for fractographic applications. Also when different methods for characterizing the images of fracture surfaces were used, it was found that images cannot be characterized by a single numerical parameter. This is due to the fact that the character of fracture surface is too multiform, complicated and variable. At least several parameters have to be used in order to represent the morphology of crack surface, being simultaneously sufficient for estimating crack growth rate.

In addition, standard methods for estimation of the fractal dimension inherently presuppose the condition of self-similarity. However, fractographic structures often do not satisfy this condition to an acceptable degree.

Because of these considerations, we decided to seek for multidimensional fractal characteristics that would be free of the presumption of self-similarity and applicable in the fractography of fatigue fractures.

\section{MATERIAL AND METHODS}

\section{FRACTOGRAPHY OF FATIGUE FRACTURES}

The general aim of fractography is to relate the morphology of a fracture surface to conditions of the fracture process (material, loading, temperature, environment, crack growth direction and rate, etc.). Within this frame, quantitative fractography uses numerical parameters to characterize morphologic features of the fracture surface. One of the most successful applications is the reconstitution of the fatigue crack growth history from the fracture surface.

All analyses of this type contain the following steps: 
- Laboratory experiments: specimens of the investigated material are loaded under the given conditions. Crack growth is recorded so that CGR can be assigned to each location of the crack surface.

- Images of crack surfaces in a selected magnification are usually created by means of a scanning electron microscope (SEM). CGR is assigned to single images.

- Using a suitable method, each image is characterized (all images in the same way) by a set of numerical parameters - feature vector.

- Feature vectors are related to CGR by means of multidimensional statistical methods or a neural network.

\section{Textural method}

Within conventional fractography, great magnifications are used (from about $1000 \times$ to $30000 \times$ ) and images are characterized by a single number - striation spacing.

In textural fractography, smaller magnifications are applied, typically $200 \times$. Images are understood as image textures. Images are characterized by a feature vector consisting of several, up to several tens, of components. It may be constructed in many ways (Lauschmann et al., 2002): from a decomposition (Fourier, Wavelet, etc.), via extracting objects and describing them by means of stochastic geometry, from a random field model (Markov, Gibbs), etc. Within this paper, several modifications of fractal geometry will be applied to compose image feature vectors.

\section{Multilinear model}

Let us assume a set of images denoted by index $i$ $=1, \ldots, n$ with assessed CGR values $v_{i}$. The image feature vectors composed of $k$ elements are $\boldsymbol{f}_{i}=\left[f_{i 1}\right.$, ..., $\left.f_{i k}\right]$. The most simple model of the relation between images and CGRs (usually taken as $\log (v)$ ) may be expressed in a multilinear form (Lauschmann et al., 2002)

$$
\log \left(v_{i}\right) \approx \sum_{j=1}^{k} c_{j} f_{i j}+c_{k+1}, i=1, \ldots, n,
$$

giving one equation for each image. The set of constants $\boldsymbol{c}=\left[c_{1}, \ldots, c_{k+1}\right]$ can be simply estimated by the least squares method.

Tests of the statistical significance of the contribution of single image features $f_{j}$ allow exclusion of those not predicating CGR. To receive valuable results, the system of equations must be strongly overdetermined: the number of equations must be much higher than the number of estimated constants $c$, i.e., the number of images $n$ must exceed the number of image features $k$ several times.

\section{Experiment}

CT-specimens (Fig. 1) of stainless steel AISI 304L were loaded by a constant cycle in tension with $P_{\min }=1450 \mathrm{~N}, P_{\max }=4850 \mathrm{~N}$, at frequencies 0.5 to 10 $\mathrm{Hz}$, at running temperature $20^{\circ} \mathrm{C}$ in a corrosive aggressive aqueous solution, denoted as B-water. The status of the process of crack growth was regularly recorded during the loading in the form of couples [number of loading cycles, crack length].

Fatigue crack surfaces were documented using SEM with magnification $100 \times, 200 \times$ and $500 \times$ (the real field of view being about $1.2 \times 0.9,0.6 \times 0.45$, and $0.24 \times 0.18 \mathrm{~mm}$, respectively). A sequence of images was located in the middle of the crack surface; the images were distanced by $0.4 \mathrm{~mm}$. The direction of the crack growth in images was bottom-up. Digital representation in $1200 \times 1600$ pixels and 256 brightness levels was used. Images were normalized to remove large-scale fluctuations of mean brightness and contrast.

Crack rates were assigned to single images from laboratory records mentioned above.
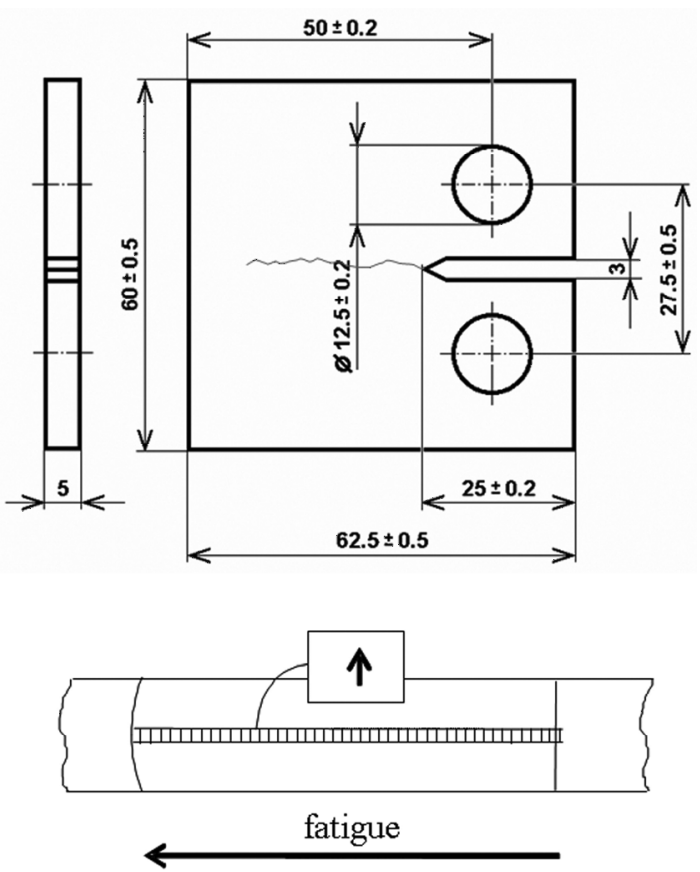

Fig. 1. Specimen for fatigue tests and locating of images in crack surface. 


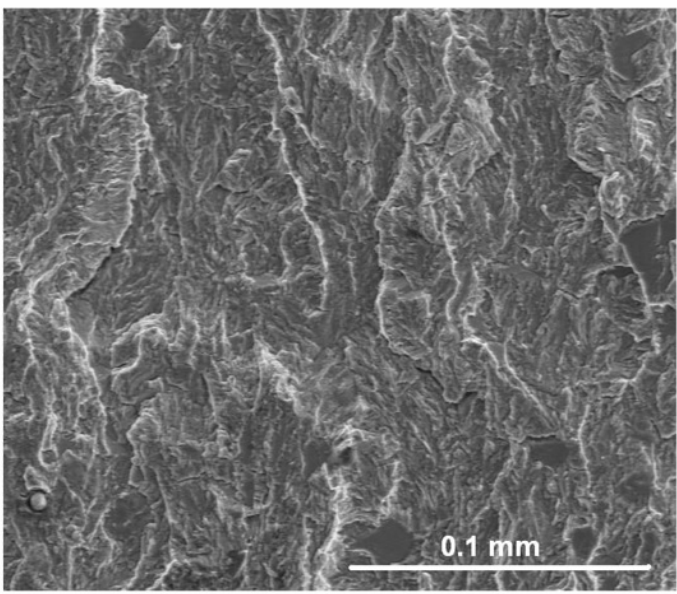

Fig. 2. Typical texture in an image of crack surface.

\section{FRACTAL ANALYSIS}

The central concept behind fractal and multifractal analysis is the notion of self-similarity, by which we understand that an object looks the same (or similar) under different magnifications. A fractal is then an object which is highly self-similar.

Fractal objects can be assigned a unique number called the fractal (Hausdorff) dimension, which is a non-integer in the case of fractals, and quantifies the self-similarity of an object.

Mathematically speaking, fractal objects are sets. This represents a binary information. A generalization of fractals are multifractals which are mathematical sets with a measure. In the case of digital images, the difference between fractals and multifractals is similar to the difference between a binary (black and white) image and a grayscale image.

Thus multifractal theory takes into account the differences between points in a set. Multifractals are described by an infinite number of generalized dimensions, in comparison with ordinary fractals characterized by one dimension. For more on multifractal theory, see Hentschel and Proccaccia, 1983, Olsen, 1995 and Bednařík, 2006.

\section{Box-counting method}

The box-counting dimension is a suitable parameter describing the fractal character of two- and threedimensional objects. In fact, it refers to one of many estimation methods defining more or less different types of fractal dimensions. However, for the sake of simplicity we will call it the "fractal dimension".

The object is covered by boxes (cubes, respectively), and their number is recorded. In a graph, we can reflect the dependence of the number of boxes (cubes) $N$ on the box (cube) side length $r$, both in logarithmic scale. Fractal dimension $D_{B}$ is defined as (Kaye, 1989)

$$
D_{B}=\lim _{r \rightarrow 0} \frac{\ln N(r)}{-\ln r} .
$$

For estimation, the dependence of $\ln N$ on $\ln r$ is fitted by a line

$$
\ln N \approx p_{1} \ln r+p_{2} .
$$

Substituting from Eq. 3 to Eq. 2 we receive

$$
D_{B}=\lim _{r \rightarrow 0} \frac{p_{1} \ln r+p_{2}}{-\ln r}=-p_{1} .
$$

The term $p_{2}$ disappears because the denominator tends to infinity.

\section{Brightness ratio}

The box-counting method for 3D may also be used for the fractal analysis of gray-scale images. In this case, two dimensions are defined by lateral coordinates in the plane of the image, with the pixel size as the standard measuring unit. The third dimension is defined by the value of brightness. As the basic measure for the third dimension, the 8-bit scale (the range of 256 brightness values) may be taken. However, with respect to the non-homogeneity of the set of dimensions, the ratio size unit / brightness unit is not obvious and its effect should be investigated.

In order to explore how the choice of units influences the results, we have introduced the brightness ratio $p_{j}$ defined by equation

$p_{j}=$ number of brightness levels assumed / 256.

For example, let us compare selections $p_{j}=0.25$ (6-bit brightness range corresponding with the human eye sensitivity) and $p_{j}=1$ (standard 8-bit brightness range). The spatial and brightness intervals are paired differently - the "cube height" in the second case is a quarter in comparison with the first case. Primary images quantized to $p j=1$ may be transferred to another brightness ratio by multiplying brightness with the requested value $p_{j}$.

\section{Multiparametric fractal image feature vector}

The idea is derived in a simple way from the algorithm for estimating the conventional fractal dimension. Instead of the final fractal dimension, we use its source data - the whole relation $\ln N$ to $\ln r$ (Sekerešová and Lauschmann, 2005). With a unified choice of the set of values $r$, we work with the corresponding $N(r)$ values obtained from the images. 
Fractal dimension, as a deterministic function of values $N(r)$, is inherently included in their set. However, fluctuations of values $N(r)$ along the regression line (Eq. 3),

$$
\Delta(r)=\ln N-p_{1} \ln r-p_{2},
$$

may not be fully random - they may contain valuable information on images which is lost while estimating the fractal dimension. In our case, these fluctuations sensitively reflect the value of CGR, especially for certain values of $r$.

\section{Multifractal image feature vector}

The multifractal approach does not investigate the object as a fractal described by a single fractal dimension (parameter of self-similarity) but as a more complex combination of objects with different fractal dimensions. It is thus described not by one but by an infinite number of parameters called generalized dimensions (Olsen, 1995) which are defined in a similar manner as the box-counting dimension. We divide space into boxes of side length $r$ and then

$$
\begin{aligned}
& D_{q}=\lim _{r \rightarrow 0} \frac{1}{q-1} \frac{\ln \sum b_{i}^{q}}{\ln r}, q \in \mathbb{R}, q \neq 1, \\
& D_{1}=\lim _{q \rightarrow 1} D_{q},
\end{aligned}
$$

where $b_{i}$ is the total mass (in case of images, sum of brightness values in all pixels) of the $i$-th box and the sum is over all boxes with $b_{i}>0$.

For estimation, the dependence of $\ln \sum b_{i}{ }^{q}$ on $\ln r$ is fitted by a line

$$
\ln \sum b_{i}^{q} \approx c_{q} \ln r+d_{q} .
$$

Substituting from Eq. 8 to Eq. 7 we obtain

$$
D_{q}=\frac{1}{q-1} \lim _{r \rightarrow 0} \frac{c_{q} \ln r+d_{q}}{\ln r}=\frac{1}{q-1} c_{q} .
$$

The term $d_{\mathrm{q}}$ disappears because the denominator tends to infinity.

The advantage of the multifractal approach to image analysis is that the brightness information is already taken into account in the definition and therefore we do not need to revert to box counting in 3-D, which can be computationally more complicated and requires the selection of an unphysical parameter - the brightness ratio. Also, each image is described by multiple parameters (depending on the choice of $q$ s) compared to only one parameter in the conventional fractal analysis.

\section{Multifractal fluctuation feature vector}

Similarly as with the multiparametric method, the information about the CGRs is contained not only in the values of the generalized dimension $D_{q}$ (i.e., slope of fitted line), but also in the fluctuations of the points around the fitted line. For each $q$ and $r$ we define the fluctuation feature $f(q, r)$ similarly as in (Eq. 6),

$$
f(q, r)=\ln \sum b_{i}^{q}-c_{q} \ln r-d_{q} .
$$

\section{RESULTS}

\section{APPLICATION OF MULTIPARAMETRIC FRACTAL FEATURE VECTORS}

Multiparametric fractal feature vectors were estimated by the box-counting method. They were composed of $24 N(r)$ components, corresponding to the set of $r=\{5,7,9,11, \ldots, 51\}$ units.

The multilinear regression (Eq. 1) was used to express the relation between CGR and the components of the fractal feature vector. Within all computations, 8-11 features from the total number of 24 passed the test of significance.

Examples of results are presented in Figs. 3 and 4. The purpose of the figures is to document the quality of expression of the relation between CGR and crack image. Therefore, output CGR values (estimated from images) are plotted against the input CGR values (known from experiment; it should be stressed that the aim of this analysis was not to estimate the unknown CGRs!). Results show a narrow confidence belt in the area of ideal agreement, represented by the diagonal line $y=x$.

The effect of the brightness ratio was investigated in the interval of $p_{j} \in\langle 0.1 ; 5\rangle$. The correlation coefficient between input and output crack growth rates was used as the quality criterion. The best results were achieved in different cases for brightness ratios from about 1.5 to 3.5 . For all cases studied, the mean dependence of model quality on brightness ratio is shown in Fig. 5. 


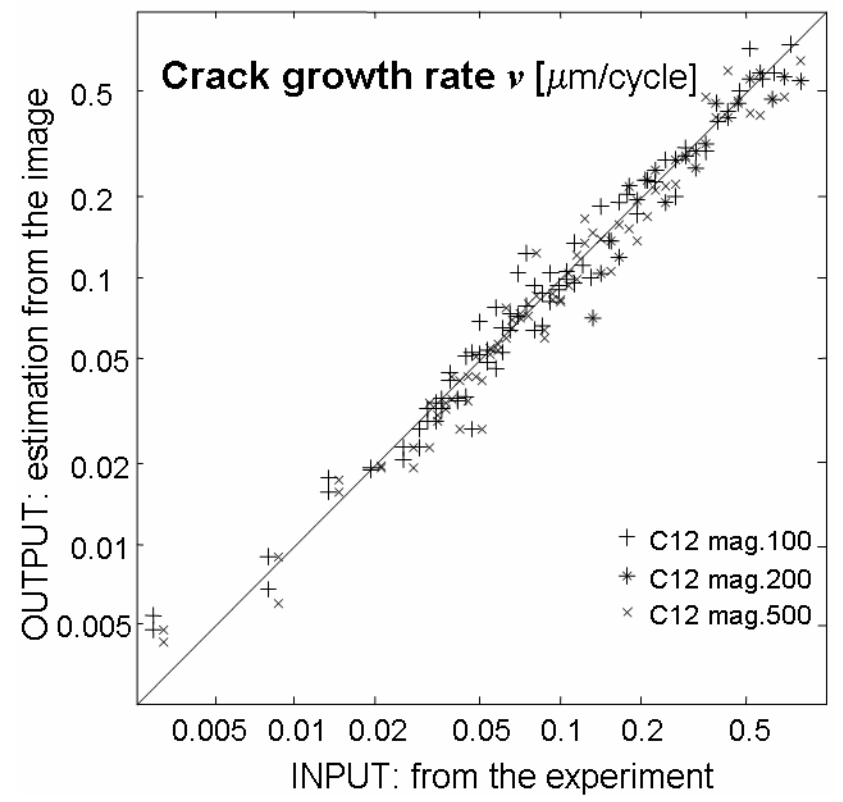

Fig. 3. Comparison of crack growth rates estimated from images of fracture surfaces with input values. One specimen, different magnifications $(100 \times, 200 \times$, $500 \times)$. One point represents one image.

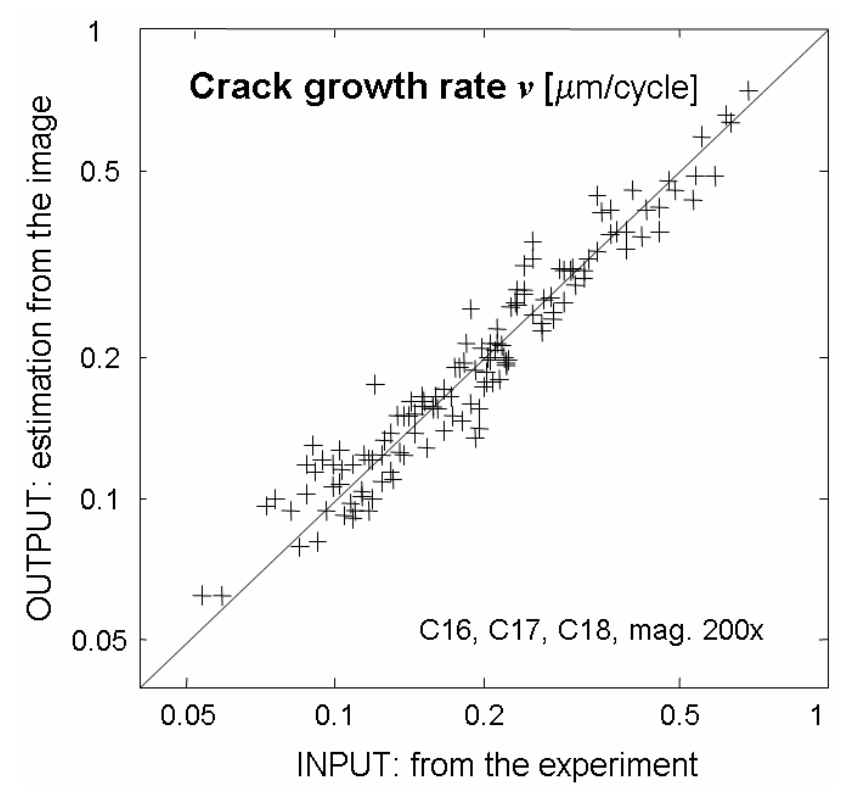

Fig. 4. Comparison of crack growth rates estimated from images of fracture surfaces with input values. Three specimens together, magnification 200×. One point represents one image.

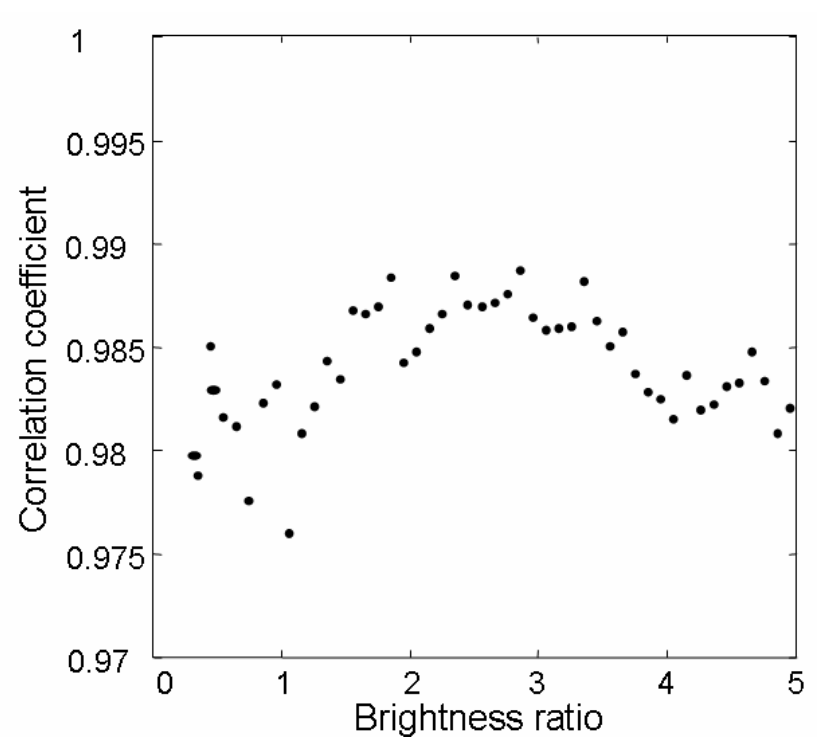

Fig. 5. The mean trend in the effect of brightness ratio.

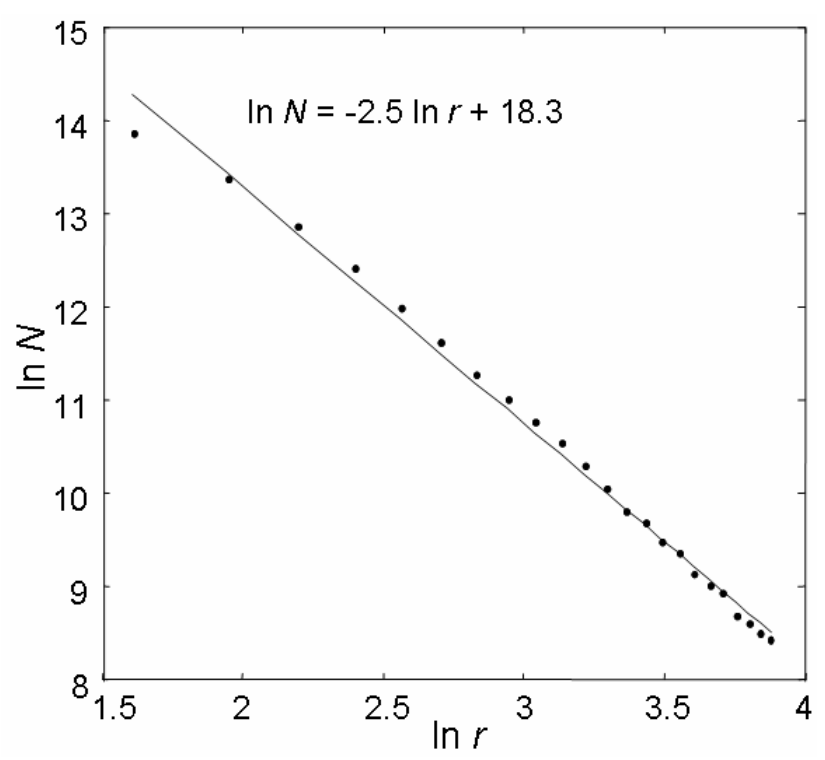

Fig. 6. An example of the relation between $\ln N$ and ln $r$ for a selected image; the interlacing line.

An example of the relation between $\ln N$ and $\ln r$ given by one image is shown in Fig. 6 together with the regression line (Eq. 3). Only this line is projected into the fractal dimension. The information enrichment through the multidimensional approach is demonstrated in Fig. 7. Fluctuations (Eq. 6), which are not reflected by the fractal dimension, are mostly dependent on CGR, therefore being a rich source of information about it. 

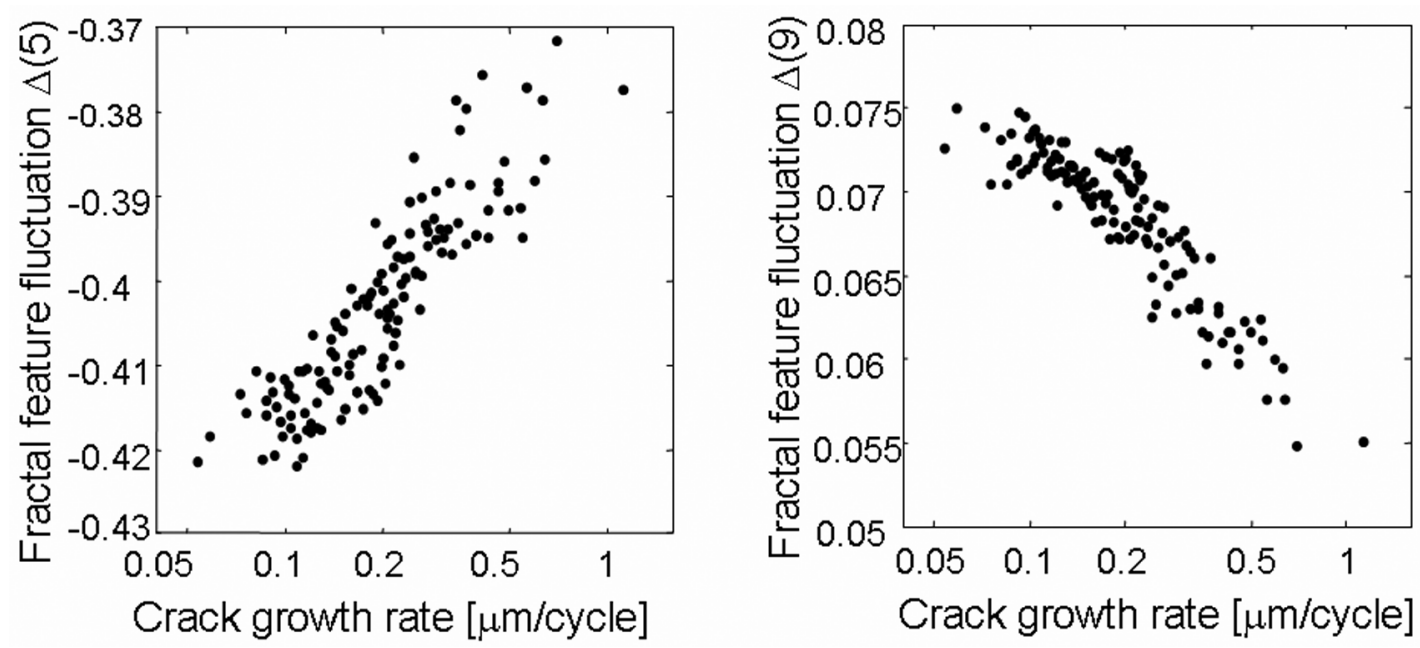

Fig. 7. Selected fractal features for $r=5$ and $r=9$ : fluctuations $\Delta(r)$, which are not reflected by fractal dimension, are a valuable source of information on CGR. Three specimens together, magn. $200 \times$ (see also Fig. 4).

\section{APPLICATION OF MULTIFRACTAL FEATURE VECTORS}

The generalized dimensions (Eq. 9) of each image were estimated for 93 parameters $q$ equidistantly spread over the interval $\langle-40,60\rangle$. An example is shown in Fig. 8. Because of the high interdependence of the features, a Principal Component Transform (PCT, Webb, 2002) was used to create uncorrelated features. A Sequential Backward Selection (SBS, Webb, 2002) algorithm was used for the selection of features. The algorithm begins with the full set of features (after PCT) and in each step the statistically least significant feature is removed. This is repeated until the desired number of features is reached, in our case 25 . The results are in Fig. 9.

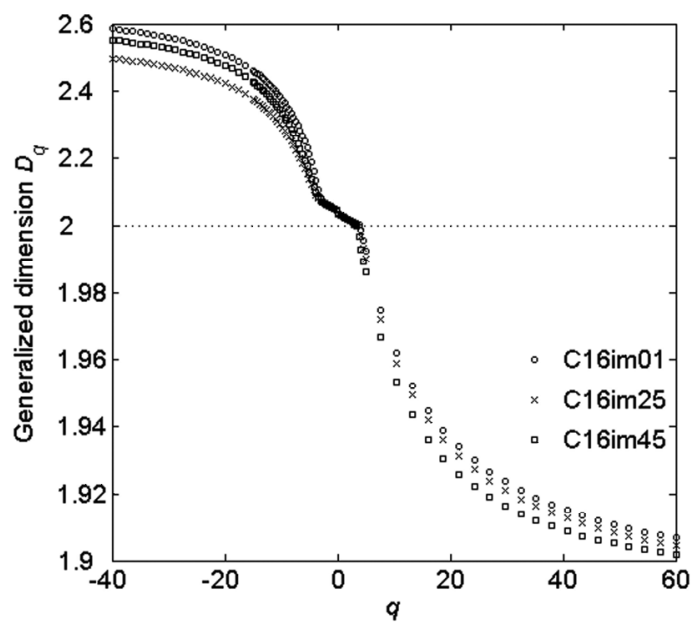

Fig. 8. Examples of calculated generalized dimensions for three selected images of specimen C16 (image 01: low CGR, image 25: middle CGR, image 45: high CGR). Magnification 200×.

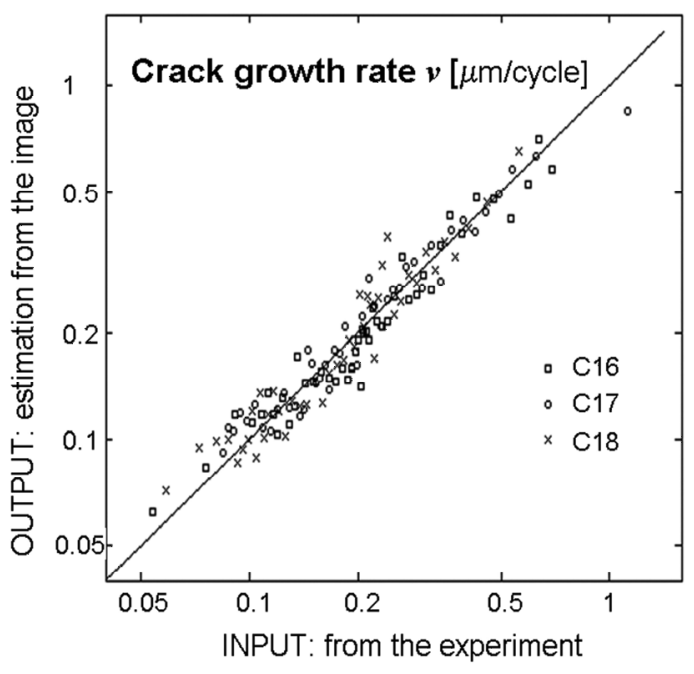

Fig. 9. Comparison of crack growth rates estimated from images of fracture surfaces with input values. Multifractal feature vector. Three specimens together, magnification $200 \times$. One point represents one image.

\section{APPLICATION OF MULTIFRACTAL FLUCTUATION FEATURE VECTORS}

Fluctuation features $f(q, r)$ (Eq. 10) were calculated for 67 values of $r$ spread over the interval $\langle 4,75\rangle$ equidistantly in logarithmical scale, and for 15 values of $q$ equidistantly spread in the interval $\langle 0,15\rangle$ giving a total of 1005 features for each image. An example of feature values is shown in Fig. 13. Because of the high number of features, a feature selection method was used. The set of features with the same $q$ is analyzed for each $q$ separately by use of the PCT and SBS algorithm as above. For the criterion of the quality of regression we take the standard deviation 


$$
\hat{\sigma}=\sqrt{\frac{1}{n-k} \sum_{i=1}^{n}\left(\hat{y}_{i}-y_{i}\right)^{2}}
$$

where $n$ is the number of images, $k$ is the number of features, $\hat{y}_{i}$ is the estimated CGR of the $i$-th image and $y_{i}$ is the measured CGR. This criterion takes into account the quality of the fit, but it also penalizes too many features. Thus the minimum of the dependence of $\hat{\sigma}$ on $k$ marks the number of features after which adding additional features is no longer effective (the gain in the quality of the fit is not sufficient). An example of the dependence of $\hat{\sigma}$ on the number of features is shown in Fig. 10. The values $\hat{\sigma}$ of these minima are compared for each $q$, the result is shown in Fig. 11. From this it can be seen that the best fit is achieved with the set of features $f(q, r)$ for $q=1.071$. This fit is illustrated in Fig. 12 for 29 selected features.

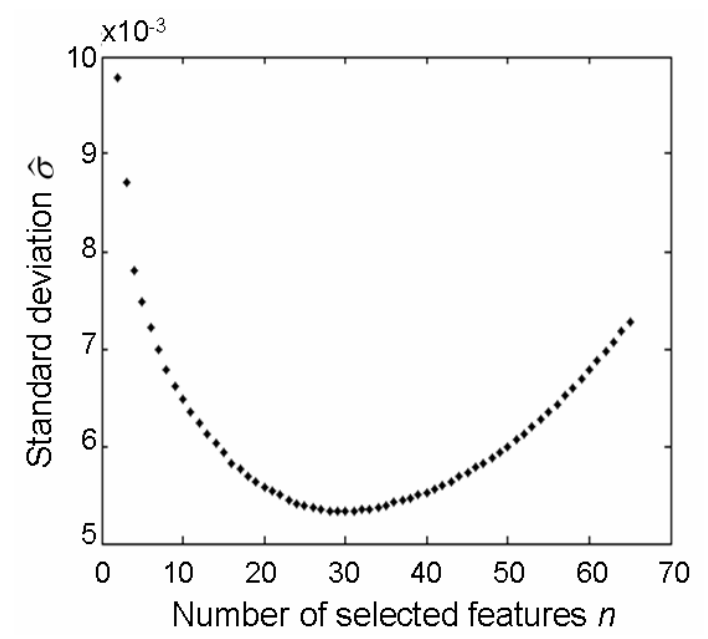

Fig. 10. An example of the dependence of the standard deviation of the regression model on the number of features $(q=1.071)$.

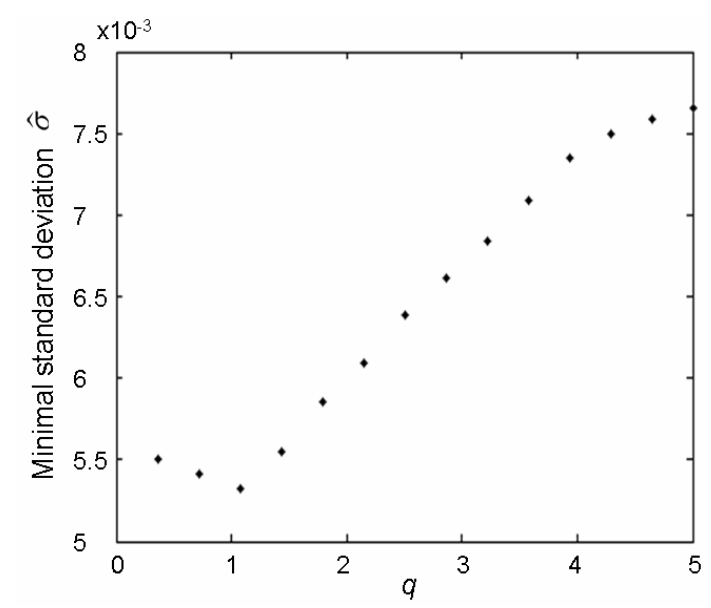

Fig. 11. The dependence of the minimal value of $\hat{\sigma}$ on $q$. Minimum corresponds to $q=1.071$.

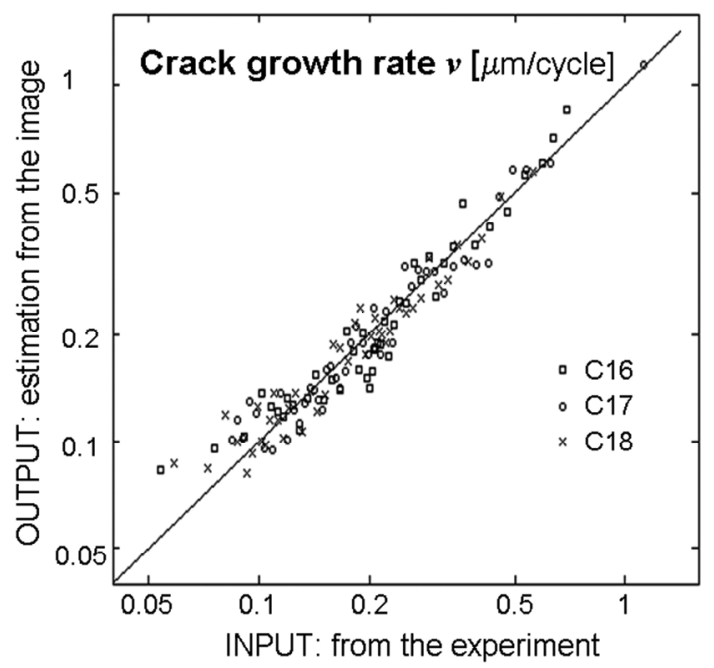

Fig. 12. Comparison of crack growth rates estimated from images of fracture surfaces with input values. Multifractal fluctuation feature vector. Three specimens together, magnification $200 \times$. One point represents one image.

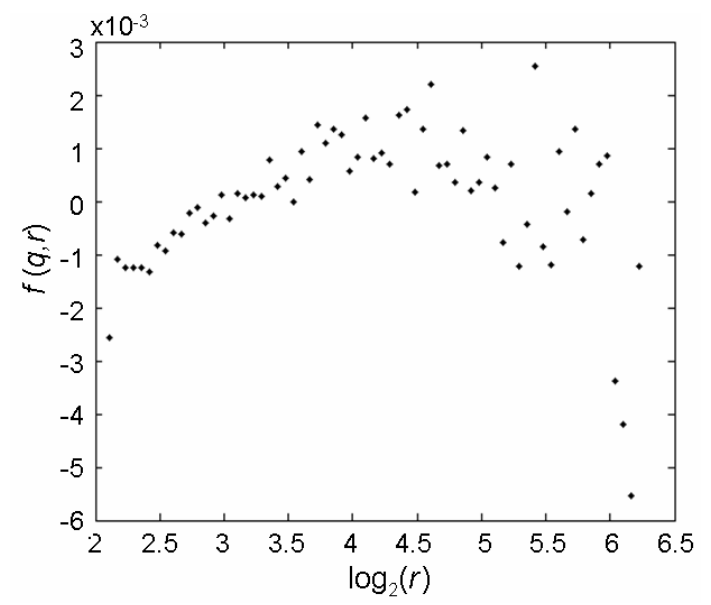

Fig. 13. An example of the values of the features $f(q, r)$ for $q=1.071$ for selected image.

\section{APPLICATION OF COMBINED MULTIFRACTAL FEATURE VECTORS}

Both the multifractal and multifractal fluctuation feature vectors give good results. However, performance can be further improved by combining these methods. The combined feature vector was composed so that the set of 29 fluctuation features (Eq. 10) was supplemented by features constructed in the same way from the 93 generalized dimensions (Eq. 9). When the generalized dimensions were processed as before by PCT and SBS, the dependence of the deviation $\hat{\sigma}$ on the number of features $k$ attained its minimum at 50 features. These 50 features were incorporated into the combined feature vector, so that it was composed of 79 features. This set was again 
processed by PCT and SBS and the final set of 25 features was selected. The best agreement between estimated and input CGRs was reached in this way, as it is evident from Fig. 14 in comparison with Figs. 4,9 and 12 .

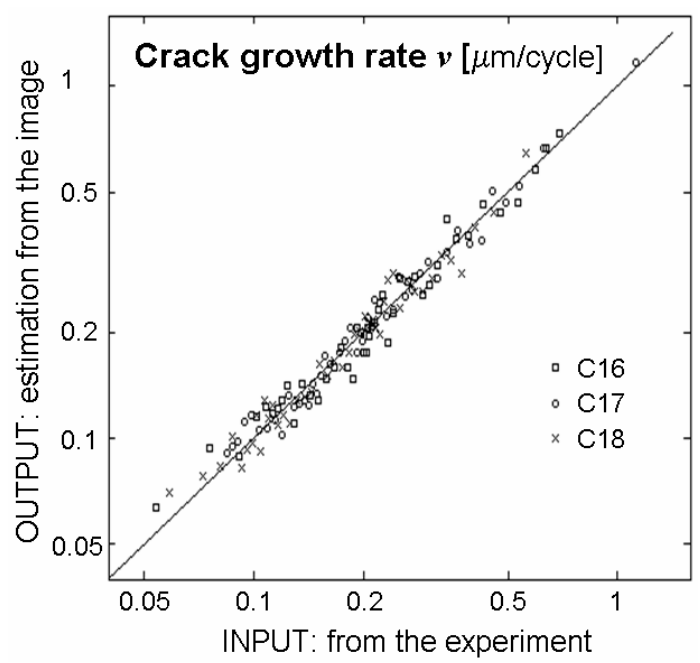

Fig. 14. Comparison of crack growth rates estimated from images of fracture surfaces with input values. Combined multifractal feature vector, 25 components. Three specimens together, magnification $200 \times$. One point represents one image.

\section{DISCUSSION}

Fractal analysis that deals with a unique characteristic - fractal dimension - is not sufficiently discriminative for the image textural analysis in fractography. Therefore, two types of fractal feature vectors composed of a set of characteristics were proposed.

Multiparametric fractal feature vector utilizes the set of values from which - within the standard fractal analysis - fractal dimension is estimated. Their fluctuations along the expected line contain a lot of information.

Multifractal theory is a generalization of fractal geometry. Instead of one fractal dimension, the object is characterized by a (generally infinite) set of generalized dimensions. They may be used as components of a fractal feature vector.

Both methods may be combined. The multiparametric approach may also be applied to multifractals, because generalized dimensions are estimated in a similar way as the standard fractal dimension. Finally, a fractal feature vector may be created as a compound of characteristics of previously mentioned types. The best results in applications are reached in the last way.
Within applications in fractography, fractal feature vectors give valuable results. Therefore, they should be further developed and used for routine fractographic analyses.

Fractal fature vectors may find use in all areas where random geometrical structures are investigated.

\section{ACKNOWLEDGEMENT}

This research has been supported by the Ministry of Education of the Czech Republic, research projects "Diagnostics of Materials" No. MSM 6840770021, and "Applied Mathematics in Technical and Physical Sciences" No. MSM 6840770010.

\section{REFERENCES}

Bednařík P (2006). Advanced methods of fractal geometry. [Research study - in Czech.] Czech Tech. Univ. Prague, Faculty of Nuclear Sci. and Physical Engng., Dept. of Mathematics.

Carpinteri A, Spagnoli A, Vantadori S (2002). An approach to size effect in fatigue of metals using fractal theories. Fatigue Fract Eng M 25(7):619-27.

Carpinteri A, Spagnoli A (2004). A fractal analysis of size effect on fatigue crack growth. Int J Fatigue 26(2):12533.

Hentschel HGE, Proccaccia I (1983). The infinite number of generalized dimensions of fractals and strange attractors. Physica D 8:435-44.

Kaye BH (1989). A random walk through fractal dimensions. New York: John Willey \& Sons.

Lauschmann H, Tůma M, Ráček O, Nedbal I (2002). Textural fractography. Image Anal Stereol 21(Suppl 1):S49-S59.

Mazancová E, Mazanec K (2000). Fractal characterization of fracture surfaces in intercritically annealed bainitic steel. In: Parilák L, ed. Fractography 2000. Stará Lesná, Slovakia: Institute of Materials Research of the Slovak Academy of Sciences, Košice, 108-15.

Mecholsky JJ, West JK, Passoja DE (2002). Fractal dimension as a characterization of free volume created during fracture in brittle materials. Phil Mag A 82(17/18): 3163-76.

Mecholsky JJ (2006). Estimating theoretical strength of brittle materials using fractal geometry. Materials Letters 60:2485-8.

Němec O, Holzmann M, Dlouhý I (2000). Fractal analysis of fracture surfaces of $\mathrm{NiCr}$ steels in transition area [in Czech]. In: Parilák L, ed. Fractography 2000. Stará Lesná, Slovakia: Institute of Materials Research of the Slovak Academy of Sciences, Košice, 116-22.

Olsen L (1995). A multifractal formalism. Adv Math 116: 82-195. 
Sekerešová Z (2004). Application of fractal geometry in textural fractography of fatigue fractures. [Research study - in Czech.] Czech Tech. Univ. Prague, Faculty of Nuclear Sci. and Physical Engng., Dept. of Materials.

Sekerešová Z, Lauschmann H (2005). A fractal study of images of fatigue fracture surfaces in relation to the crack growth rate. Juniormat '05. Brno, Czech Rep., 31-4.
Webb AR (2002). Statistical pattern recognition. Chichester: John Wiley \& Sons.

Wiencek K, Czarski A (2000). Fractal characterization of fracture surfaces. In: Wojnar L and Rozniatowski K, eds. Int. Conf. on Stereology and Image Analysis in Material Science. Kraków, Poland: Polish Society for Stereology, 427-30. 